\title{
Distribución geográfica y ecológica de Ipomoea (Convolvulaceae) en el estado de Michoacán, México
}

\section{Geographical and ecological distribution of Ipomoea (Convolvulaceae) in Michoacán State, Mexico}

\author{
Josiani Alcántar-Mejía ${ }^{1}$, Eleazar Carranza-González², Gabriela Cuevas-García ${ }^{3}$ y Eduardo Cuevas-García ${ }^{1 凶}$

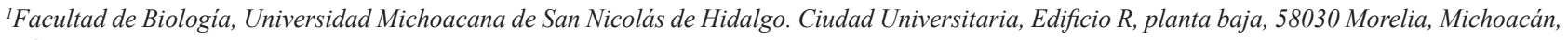 \\ México. \\ ${ }^{2}$ Instituto de Ecologia, A.C. Av. Lázaro Cárdenas 253, 61600 Pátzcuaro, Michoacán, México. \\ ${ }^{3}$ Centro de Investigaciones en Geografia Ambiental, UNAM. Antigua Carretera a Pátzcuaro 8701, Ex-hacienda de San José de la Huerta, 56190 \\ Morelia, Michoacán, México. \\ \ecuevas@ecologia.unam.mx
}

\begin{abstract}
Resumen. De los 2663 géneros de plantas vasculares registrados para México, Ipomoea (Convolvulaceae) ocupa el décimo lugar en número de especies y es el más diverso en Michoacán, donde se estudió su distribución geográfica y la relación de su riqueza con factores ambientales. En este trabajo se registraron 75 especies, lo que representa alrededor del 44\% de las registradas para México, a pesar de que Michoacán ocupa sólo el 3\% del área total nacional. Mediante revisión de 4 herbarios nacionales (EBUM, IEB, ENCB y MEXU) y exploraciones en campo, se obtuvieron 552 registros georreferenciados de Ipomoea. El estado se dividió en celdas de 15' $\times 15^{\prime}$ para ubicar las recolectas y realizar un mapa de riqueza de especies. Se exploró la relación entre la riqueza específica por cuadro y la altitud, precipitación, temperatura, tipo de vegetación y tipo de suelo. El noreste de Michoacán presentó un gran número de registros y una alta riqueza de especies; el suroeste fue la región con menos registros, pero también mostró una alta riqueza específica. Respecto a la temperatura media y precipitación, el mayor número de especies se encontró entre los 15 y $30^{\circ} \mathrm{C}$ y entre 899 y 1199 mm, respectivamente. En el gradiente altitudinal la distribución de especies fue bimodal, con un pico entre los 0 y 299 m y otro entre 1800 y 2099 m. Se encontró afinidad de estas plantas con el bosque tropical caducifolio, el pastizal secundario y los suelos de tipo litosol, vertisol y luvisol.
\end{abstract}

Palabras clave: gradientes altitudinales, precipitación, Sistemas de Información Geográfica, temperatura.

\begin{abstract}
Absctract. Of the 2663 genera of vascular plants in Mexico, Ipomoea (Convolvulaceae) ranks 10th in number of species, and it is the most speciose genus in Michoacán. With 75 species recorded from the state, Michoacán contains approximately $44 \%$ of the species reported from Mexico, this despite that the state represents only $3 \%$ of the country's surface area. We studied the geografic distribution and the relation between species richness and environmental factors within Michoacán. Through the examination of the major Mexican herbaria, as well as field exploration, we obtained 553 georeferenced specimens. The state was divided into $15 \mathrm{~km}^{2}$ quadrants in order accommodate the collections, and a map was produced to demonstrate species richness. The relation between species richness and altitude, precipitation, temperature, soil, and vegetation type was explored. Northeastern Michoacán had the greatest number of specimens, as well as the highest species richness. On the contrary, the southwestern portion of the state had the fewest specimens, but also had high species richness. With respect to mean temperature and precipitation, the greatest number of species was found to occur between 15 to $30^{\circ} \mathrm{C}$ and 899 to $1199 \mathrm{~mm}$, respectively. The altitudinal gradient of the distribution was bimodal, with one peak between 0 to $299 \mathrm{~m}$ and another between 1800 and $2099 \mathrm{~m}$. A preference was found for tropical deciduous forest and secondary grasslands, as well as for litosol, vertisol, and luvisol soils.
\end{abstract}

Key words: altitudinal gradients, Geographic Information System, precipitation, temperature.

\section{Introducción}

México se ubica entre los países con mayor riqueza florística (Rzedowski, 1978) acompañada de un número

Recibido: 01 junio 2011; aceptado: 28 marzo 2012 importante de endemismos. Esta situación ha motivado la realización de diversos estudios enfocados a estimar la biodiversidad del país (Rzedowski 1991; Villaseñor, 2003; Villaseñor et al., 2005), conocer la flora de regiones específicas (Medina et al., 2000; Trejo 2005; Cué Bär et al., 2006a; Ramírez et al., 2010), la diversidad de especies a distintos niveles taxonómicos (Valencia, 2004; Rzedowski 
et al., 2005; Cuevas et al., 2008) y los factores (actuales e históricos) que puedan explicar tales patrones (Lindenmayer et al., 1991; Espinosa y Ocegueda, 2008).

Desde el punto de vista florístico, el estado de Michoacán es uno de los más relevantes del país, lo que en gran medida se debe a su gran variedad de ambientes y accidentada topografía (Carranza, 2008). A pesar de que no se cuenta con un dato exacto, se estima que Michoacán podría albergar unas 5000 especies de angiospermas pertenecientes a un poco más de 1000 géneros de 185 familias (Carranza, 2005a). Dentro de esta diversidad, actualmente Ipomoea (Convolvulaceae) es el género con mayor número de especies en el estado, seguido por Salvia (Lamiaceae) (Carranza, 2005b; Cornejo-Tenorio e Ibarra-Manríquez, 2011), y es el décimo en el país con 162 especies (Villaseñor, 2004), aunque en la actualidad se estiman 168 (E. Carranza, datos no publicados). A escala mundial, el género está formado por 600 a 700 especies (Austin y Huáman, 1996), las cuales presentan una distribución pantropical, con algunos taxones en latitudes templadas. Sin duda es el género con mayor riqueza en Convolvulaceae y también el más diverso en cuanto a formas de crecimiento se refiere (Austin y Pedraza, 1983; McDonald, 1991; Murguía et al., 1995). Ipomoea agrupa representantes de los más conspicuos y llamativos de la flora mexicana (Fig. 1), e incluye desde pequeñas especies de hierbas (erectas, postradas o volubles) hasta grandes lianas o árboles. Además, habita en toda una gama de condiciones climáticas, edafológicas, topográficas y de tipos de vegetación (Carranza, 2004). Sin embargo, a pesar de la relevancia que tiene este género en México, y en particular en Michoacán (Carranza, 2008), no existen trabajos que hayan documentado la distribución de sus especies en el estado ni sus posibles relaciones con algunos factores ambientales, lo cual es el objetivo central de este trabajo.

\section{Materiales y métodos}

Zona de estudio. El estado de Michoacán de Ocampo, con una superficie de $58994 \mathrm{~km}^{2}$ se localiza en la región Centro Occidente de México, entre las coordenadas 20²3'37" - 17 53 '50" N, 10003'32" - 10344'49” O (Durán y Sevilla, 2003). Michoacán está comprendido en 2 de las 15 provincias fisiográficas que conforman la República Mexicana (INEGI, 2005): la Sierra Madre del Sur (SMS; con 4 subprovincias y una discontinuidad) y

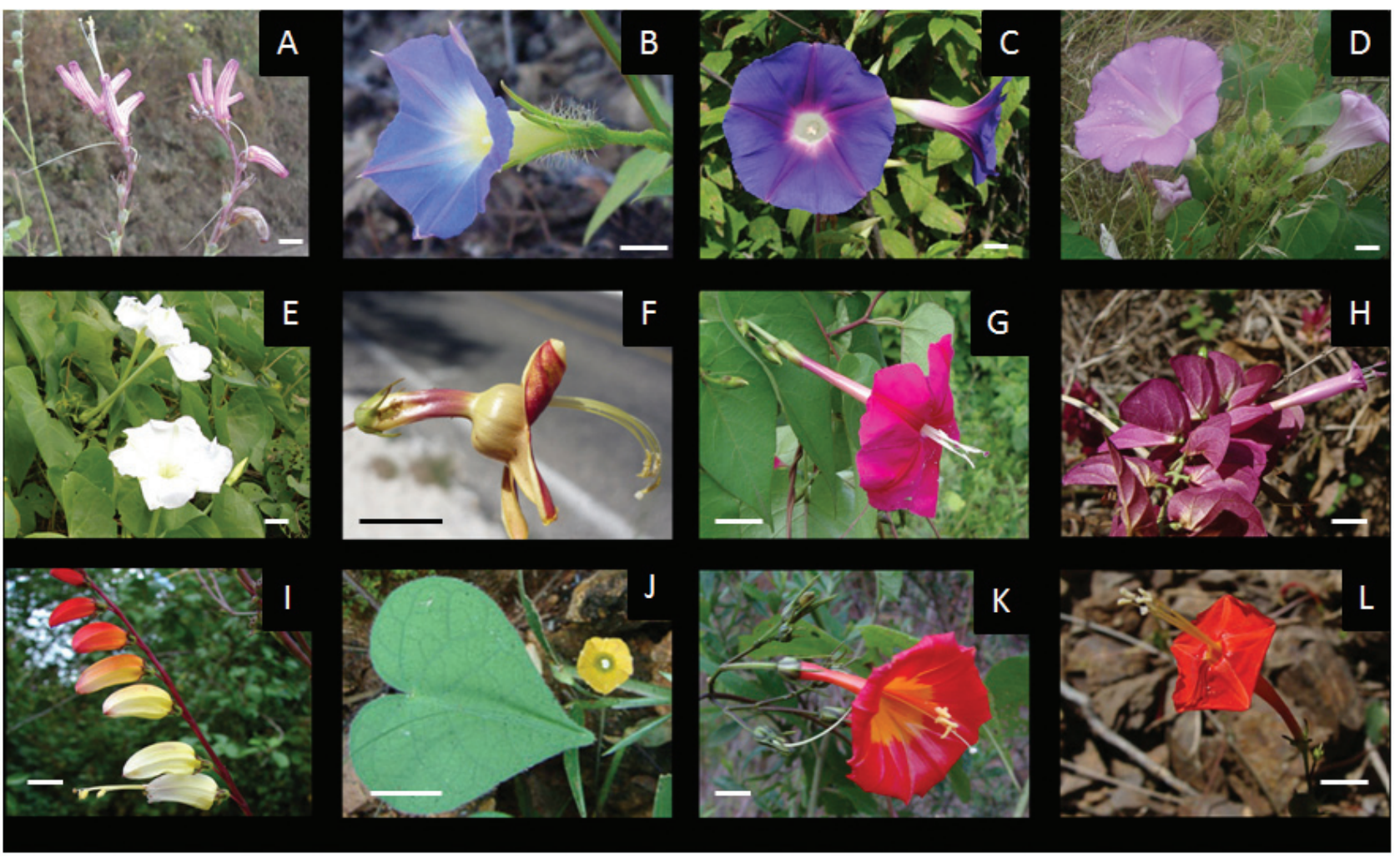

Figura 1. Ejemplos de la diversidad floral de especies del género Ipomoea en Michoacán. A, I. gloverae, una de las 2 especies endémicas de Michoacán; B, I. barbatisepala; C, I. tacambarensis; D, I. alba; E, I neei; F, I. dumosa; G, I. lobata; H, I. minutiflora; I, I. funis; J, I. crinicalyx; K, I. bracteata; L, I. hederifolia. Escala: $1 \mathrm{~cm}$. 
el Sistema Volcánico Transversal, también denominado Eje Neovolcánico (EN; con 7 subprovincias) (Fig. 2). La accidentada topografía y el amplio intervalo altitudinal de Michoacán, desde el nivel del mar, hasta los $3840 \mathrm{~m}$ en el volcán de Tancítaro, son los principales factores para explicar los diferentes climas de la entidad, siendo los principales tipos (Antaramián, 2005): el tropical lluvioso, con lluvias en verano (Aw), el seco o árido (BS), el templado con lluvias en verano $(\mathrm{Cw})$ y el templado con lluvias todo el año (Cf). Las lluvias se presentan de julio a noviembre en casi todo el estado, aunque existen zonas que reciben precipitación todo el año. El valor promedio anual de precipitación es de $961 \mathrm{~mm}$; la precipitación mínima no supera los $600 \mathrm{~mm}$ al año, y se presenta en la depresión del río Tepalcatepec; la máxima alcanza los $1600 \mathrm{~mm}$ anuales en los alrededores de Uruapan (Antaramián, 2005). De acuerdo con Carranza (2005a), Michoacán presenta 11 tipos de vegetación (bosque de coníferas, bosque de encino, bosque mesófilo de montaña, bosque tropical caducifolio, matorral subtropical, bosque espinoso, bosque tropical subcaducifolio, vegetación acuática y subacuática, palmar, pastizal y vegetación de dunas costeras). Finalmente, los tipos de suelo quedan comprendidos en 14 unidades, siendo los más importantes por la superficie que ocupan los vertisoles, litosoles o leptosoles, luvisoles, andosoles, regosoles, feozem y acrisoles (Durán y Sevilla, 2003).

Recopilación de la información. Se revisaron los ejemplares del género Ipomoea recolectados en Michoacán y depositados en el Herbario de la Escuela de Biología de la Universidad Michoacana de San Nicolás de Hidalgo (EBUM), Herbario del Instituto de Ecología A. C., Centro Regional del Bajío (IEB), Herbario Nacional del Instituto de Biología, de la Universidad Nacional Autónoma de México (MEXU) y el Herbario de la Escuela Nacional de Ciencias Biológicas, del Instituto Politécnico Nacional (ENCB). Para verificar la correcta identificación de los ejemplares, se llevó a cabo una revisión detallada de cada ejemplar, mediante la clave dicotómica de Ipomoea (Carranza, 2008) y la depuración de algunas sinonimias detectadas. Los criterios que debía cumplir cada ejemplar para ser incorporado en la base de datos fueron los siguientes: a) nombre científico; b) localidad de recolecta completa o coordenadas geográficas; c) altitud y $d$ ) tipo de vegetación. Los ejemplares que carecían de localización geográfica y/o altitud, fueron georreferenciados con base en la localidad de recolecta mediante el programa Google Earth. Los ejemplares sin localidad de recolecta no fueron incluidos en la base de datos. Se excluyeron para el análisis los registros de las especies cultivadas (I. cairica e $I$. carnea). Se realizaron 5 salidas de exploración a través del territorio michoacano, principalmente en zonas que se sabía presentaban una alta diversidad de especies de Ipomoea y en lugares en los que las recolectas han sido escasas (Carranza, 2008). Los ejemplares recolectados durante dichas exploraciones fueron depositados en el herbario del IEB.

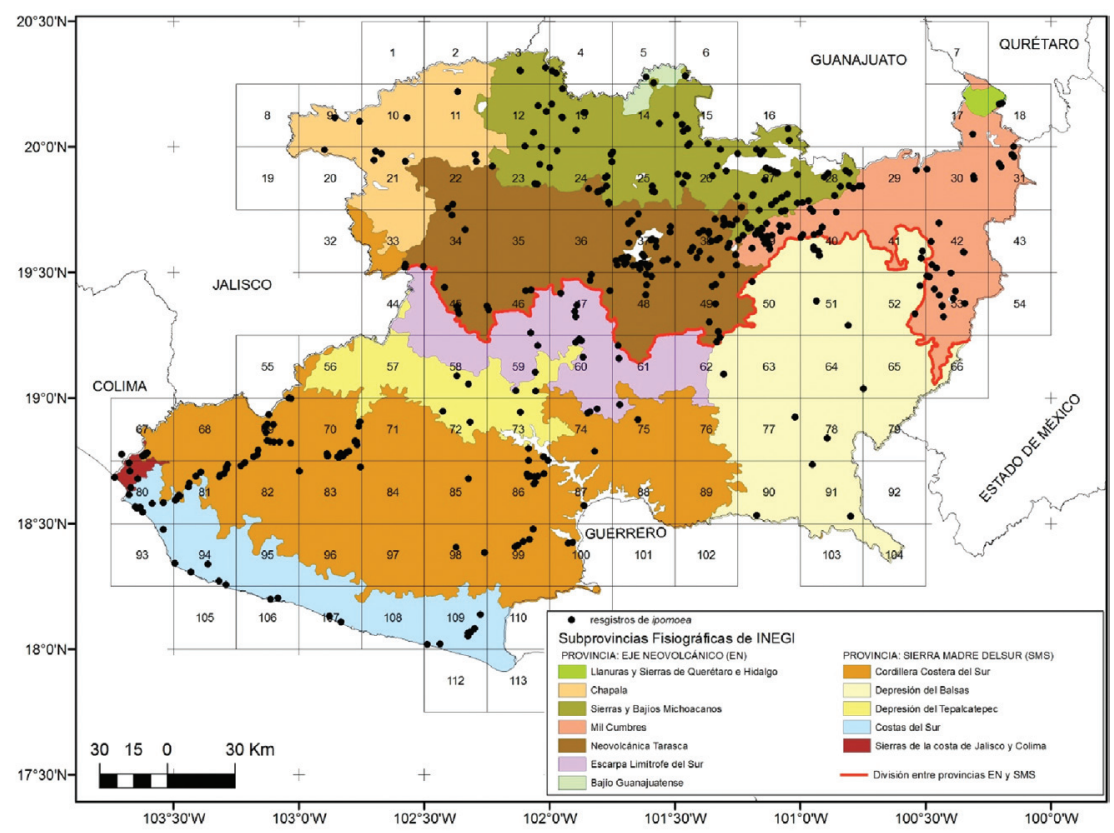

Figura 2. Regiones fisiográficas de Michoacán donde se muestra la retícula de $15^{\prime} \times 15^{\prime}$ y la distribución de los registros de Ipomoea. Línea roja continua, límite entre el Eje Neovolcánico (EN) y la Sierra Madre del Sur (SMS). 
Elaboración de mapas de distribución. Mediante el uso del programa ArcGIS (Versión 9.3) el estado se dividió en 113 cuadros o unidades de clasificación geográfica (UCG) de $15^{\prime} \times 15^{\prime}$ que equivalen aproximadamente a $735 \mathrm{~km}^{2}$ cada uno (Murguía y Rojas, 2001; Ramos-Vizcaíno et al., 2007; Figura 2). Posteriormente, a partir de las coordenadas geográficas de los puntos de recolecta, se elaboraron mapas de distribución geográfica y de riqueza de especies utilizando el mismo programa.

Relación con factores ambientales. Mediante la exploración visual de los datos graficados se determinó la relación de la distribución de la riqueza de especies de Ipomoea con algunas variables ambientales (altitud, temperatura, precipitación, tipos de vegetación y tipos de suelo). La información de la temperatura y precipitación media anual de las localidades de recolecta de los ejemplares se obtuvo a partir de la base de datos WorldClim (http://www.worldclim.org/; Hijmans et al., 2005). Los datos sobre el tipo de suelo se obtuvieron de la carta edafológica escala 1:250 000 del INEGI (2005) y los tipos de vegetación de Carranza (2005b).

\section{Resultados}

Se obtuvo un total de 637 registros de recolectas de Ipomoea para el estado de Michoacán, de los cuales 85 se excluyeron por no cumplir con los requisitos previamente establecidos. De los registros analizados, 512 se obtuvieron de datos de herbarios y 40 fueron resultado de las recolectas en campo de este estudio, los cuales permiten listar un total de 75 especies y 2 variedades de I. orizabensis (Apéndice). Ipomoea hederacea e I. aff. proxima son nuevos registros para el estado, mientras que I. fissifolia e I. gloverae son endémicas de Michoacán. En el caso de I. barbatisepala, I. decemcornuta, I. lambii, I. x leucantha e I. lindenii, únicamente se conocen de una localidad en Michoacán. Finalmente, a pesar de que se encontraron registros de herbario de I. populina e I. umbraticola, hasta el momento no se ha comprobado su presencia en el estado, debido a que la complejidad taxonómica de ambas especies hace difícil su determinación.

Distribución, riqueza de especies y números de registros. La especie mejor representada fue Ipomoea orizabensis var. orizabensis, con 60 registros equivalentes a $11 \%$ del total analizados, seguida por $I$. purpurea (46) e $I$. murucoides (44). Por el contrario, hay 32 especies con 3 o menos registros en las colecciones (Apéndice). Respecto a la forma de crecimiento, la mayoría de las especies son herbáceas trepadoras (45), 13 trepadoras leñosas, 3 arbóreas, y algunas herbáceas erectas y rastreras (Apéndice). De las 113 celdas en las que se dividió el estado, 36 (32\%) no tuvieron registros (Fig. 3). Respecto al número de especies, en 6 celdas (5\%) se obtuvieron más
Cuadro 1. Número de especies de Ipomoea registradas en distintos estados del país. En todos los casos se excluyeron las especies cultivadas

\begin{tabular}{lcc}
\hline Estado & $\begin{array}{c}\text { Número } \\
\text { de } \\
\text { especies }\end{array}$ & Referencia \\
\hline Aguascalientes & 16 & García et al. (1999) \\
Guanajuato & 23 & Carranza (1998) \\
Jalisco & 72 & Ramírez et al. (2010) \\
Oaxaca & 75 & García-Mendoza y Meave (2011) \\
Veracruz & 53 & Mc Donald (1994) \\
Michoacán & $\mathbf{7 5}$ & este estudio \\
\hline
\end{tabular}

de 11 especies en cada una; 2 de las celdas corresponden a la región noreste correspondiente al Eje Neovolcánico (EN), lo que la hace la mejor representada en número de registros (Fig. 2). La zona suroeste, con 4 celdas donde se registraron más de 11 especies, es una de las de mayor riqueza, a pesar de tener un menor número de recolectas (Fig. 2 y 3). Posteriormente, al comparar las especies del EN con las de la sierra Madre del Sur (SMS) se encontró que 35 especies (46\%) presentan una distribución limitada a una región en particular; de las cuales, 13 especies se encuentran exclusivamente en el EN y 22 en la SMS (Apéndice) y 5 especies únicamente se distribuyen en el límite entre ambas zonas, correspondiente en este caso a las subprovincias de la Escarpa Limítrofe del Sur, Depresión del Balsas y Depresión del Tepalcatepec (Apéndice).

Relación con factores ambientales. En relación con la altitud, el mayor número de especies se encontró en los intervalos de 0-299 m y de $1800-2099 \mathrm{~m}$, con 32 y 31 especies, respectivamente. También se puede observar que para el primero de estos intervalos existe un menor número de registros en comparación con el segundo. Finalmente, se observa que por arriba de los $3000 \mathrm{~m}$ no se establecen especies de este género (Fig. 4A).

Con respecto a los valores de precipitación, las especies de Ipomoea se encontraron desde los 600 a $1500 \mathrm{~mm}$ y la mayor riqueza entre los 900 y $1049 \mathrm{~mm}$, con 49 especies (Fig. 4B). En relación con la temperatura media anual, el mayor número de especies (43) se encontró entre los $25 \mathrm{y}$ $30^{\circ} \mathrm{C}$ y el menor, con sólo 8 especies, entre los 10 y $14^{\circ} \mathrm{C}$ (Fig. 4C). En cuanto al tipo de suelos, el litosol es donde se registró el mayor número de especies (43), seguido por el vertisol (37) y luvisol (35).

Por último, al analizar la distribución de la riqueza en relación con el tipo de vegetación se encontró una gran afinidad de Ipomoea con el bosque tropical caducifolio (49 especies). En contraste, el bosque de pino, el bosque mesófilo de montaña y las dunas costeras presentaron la menor riqueza con 6,2 y 1 especies, respectivamente (Fig. 4D). 


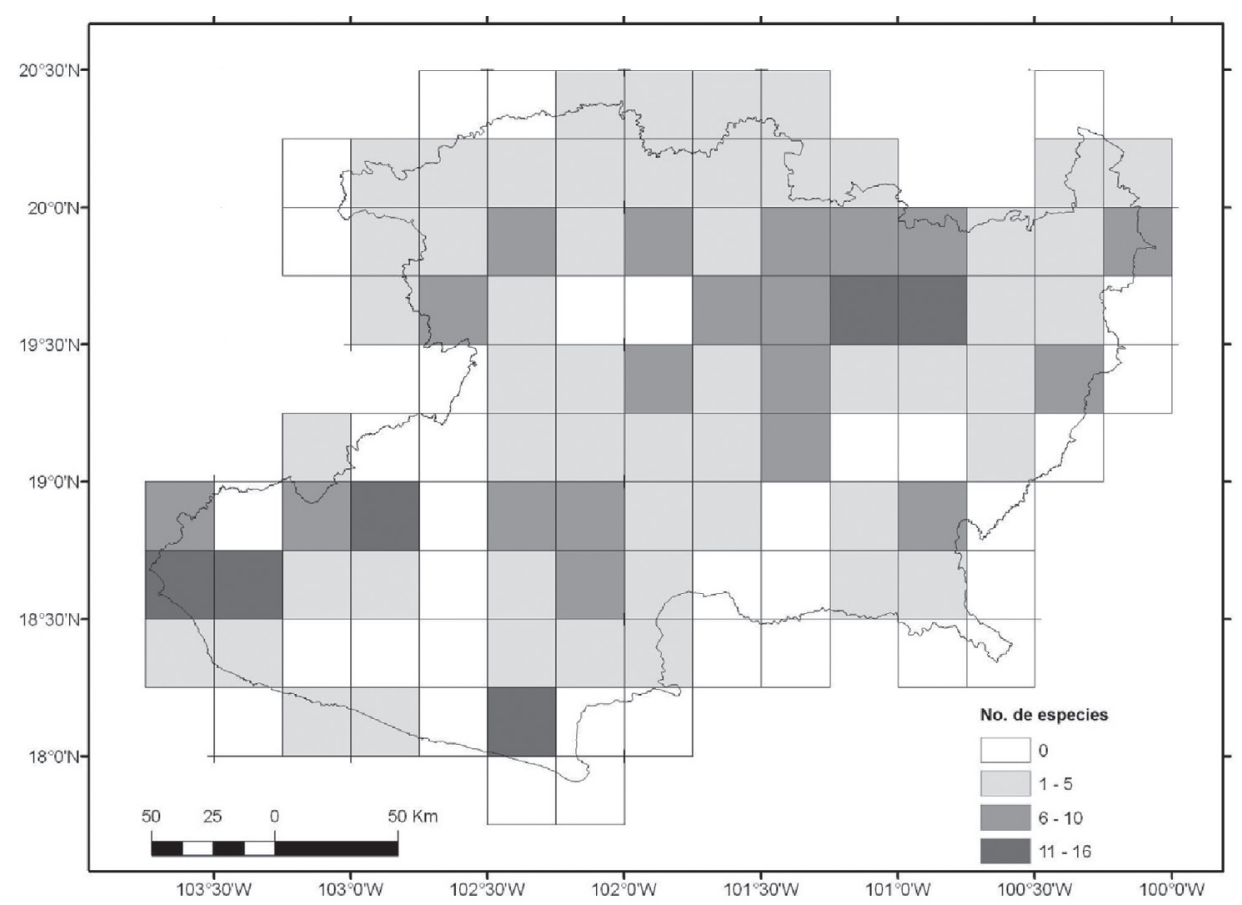

Figura 3. Estado de Michoacán con retícula de $15^{\prime} \times 15^{\prime}$, que muestra los 113 cuadros en los que se dividió el estado y la riqueza de especies por cuadro.

\section{Discusión}

Distribución, riqueza de especies y números de registros. Es relevante que en este trabajo se registraran 75 especies de Ipomoea, que representan el $44 \%$ del total conocido para el género a nivel nacional; de éstas, 25 son endémicas de México (Carranza, 2008). Estos resultados adquieren mayor relevancia si se considera que Michoacán representa únicamente el 3\% de la superficie del país (INEGI, 2010). En lo que se refiere a la riqueza de especies conocida hasta el momento en México, Michoacán ocupa el segundo lugar, únicamente por debajo de Jalisco (Cuadro 1). Otro aspecto relevante fueron los 2 nuevos registros (I. hederacea e $I$. aff. proxima) para Michoacán, los cuales se suman a la lista proporcionada por Carranza (2008). Asimismo, hay 5 especies que se han encontrado en localidades de estados cercanos a Michoacán; las cuales, por la afinidad con el tipo de vegetación podrían ser registradas en el futuro ( $I$. imperati (Vahl) Griseb., I. bombycina (Choisy) Benth. et Hook. f. e I. gesnerioides J. A. McDonald en Guerrero; I. laeta A. Gray en Jalisco; I. chamelana J. A. McDonald en Guerrero y Jalisco).

Lo anterior apunta a la necesidad de continuar con las exploraciones de campo en diferentes estaciones del año y en las regiones que presentaron un menor número de registros, así como en las regiones de una alta diversidad, con la finalidad de actualizar los datos de riqueza de especies como recientemente ocurrió con el género Salvia en Michoacán (Cornejo-Tenorio e Ibarra-Manríquez, 2011).

Además de las 2 especies registradas como endémicas de Michoacán (I. fissifolia e I. gloverae), hay otras 5 de distribución restringida a Michoacán que sólo se han registrado para otro estado del país (I. crinicalyx, Yucatán; I. lambii, Nayarit; I. pruinosa, Guerrero; I. spectata, Jalisco; I. tacambarensis, Oaxaca). Una situación similar se encontró en la distribución de las especies arbóreas de Michoacán (Cüe-Bar et al., 2006a), lo que pone de manifiesto las similitudes florísticas entre los estados al encontrarse dentro de provincias fitogeográficas comunes (sensu Rzedowski, 1978).

Por otra parte, hay 6 especies que únicamente se han recolectado en una localidad del estado (Apéndice). A pesar de que existen registros en otras regiones dentro y fuera del país, en las exploraciones de los últimos años no se han encontrado poblaciones de estas especies. Un caso particular es el de I. fissifolia, registrada como endémica de Michoacán, cuya identidad y existencia se conoce por el ejemplar tipo descrito por McPherson (1980) y de un registro de 1992. Y respecto a I. populina e I. umbraticola, se encontró una recolecta de cada especie sin que se haya podido corroborar su presencia en el estado. Al ser dudosa su identidad, se optó por no incluirlas en el listado final.

Se observa que el norte del territorio michoacano ha sido el más explorado, lo que se refleja en un mayor 

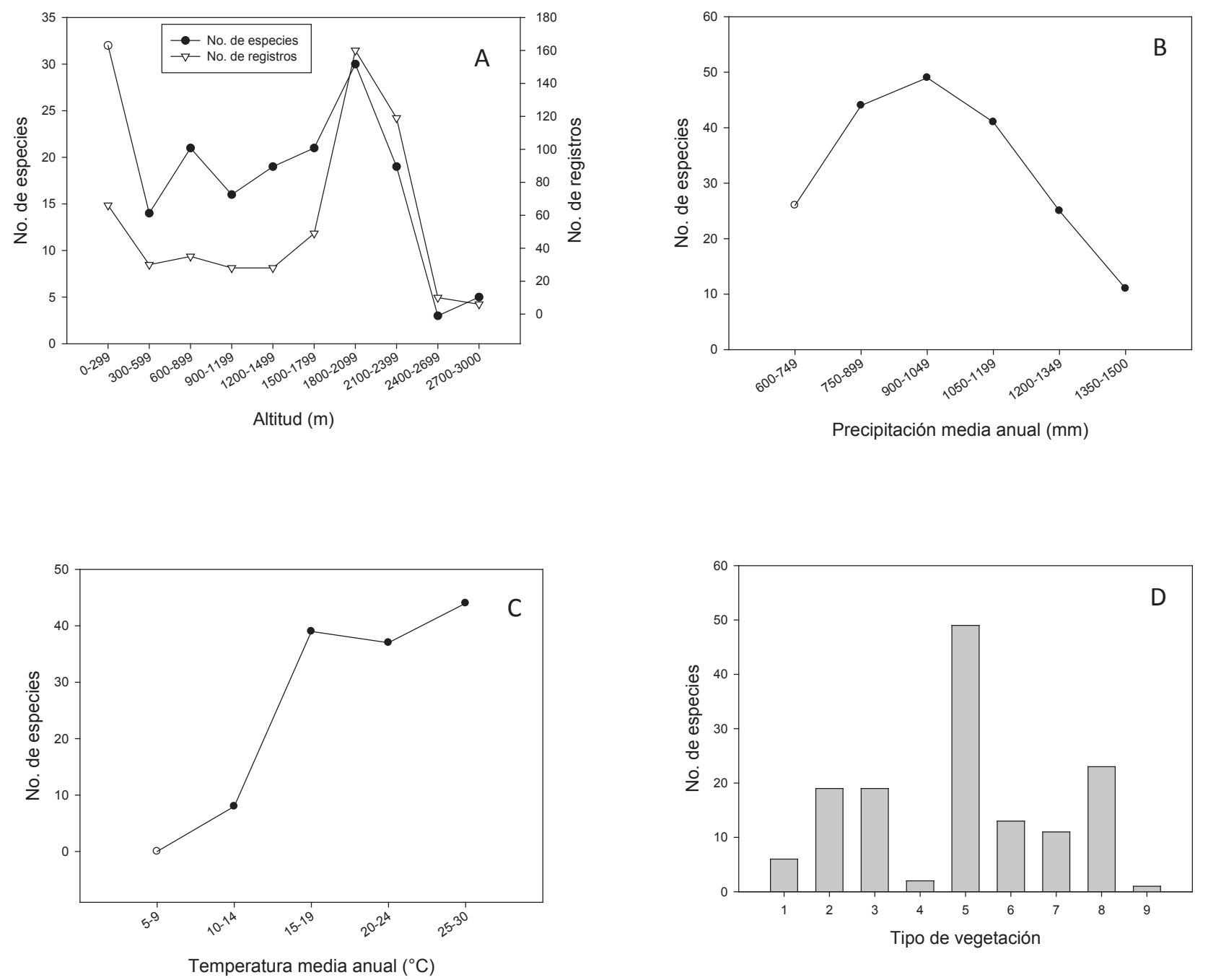

Figura 4. Número de especies en relación con: A, altitud; B, precipitación; C, temperatura, y D, Tipos de vegetación (1, bosque de pino; 2, bosque de encino; 3 , bosque de pino-encino; 4 , bosque mesófilo de montaña; 5 , bosque tropical caducifolio; 6 , bosque tropical subcaducifolio; 7, matorral subtropical; 8, pastizal secundario; 9, vegetación de dunas costeras).

número de recolectas (Fig. 2). Una situación inversa acontece con la flora de la SMS que ha sido poco explorada, tanto por su topografía accidentada como por la falta de seguridad en la zona (Fig. 2; Carranza, 2005a); a pesar de lo cual, fue una de las 2 regiones con mayor riqueza de especies de Ipomoea junto con el noreste del EN. Resultados similares están documentados para la flora arbórea y el género Salvia en el estado (Cüe-Bar et al., 2006a, Cornejo-Tenorio e Ibarra-Manríquez, 2011). Es relevante el haber encontrado que numerosas especies se distribuyen de manera restringida en el EN o en la SMS (Apéndice), lo que podría estar relacionado con factores altitudinales y de precipitación que limiten su distribución.
El alto número de registros de I. orizabensis var. orizabensis, planta abundante en encinares y pinares en el norte de Michoacán, se debe sin duda, a que esta zona es la mejor recolectada del estado y a sus grandes flores, su vistoso color y amplia distribución geográfica. Algo similar ocurre con I. purpurea, que es además una especie colonizadora de ambientes perturbados y con una distribución geográfica muy amplia. Tales características contrastan con las de otras especies, como I. minutiflora o I. costellata, que fácilmente pasan desapercibidas por el pequeño tamaño de sus corolas y de la planta en general. Es probable que en este estudio se haya subestimado la distribución de las especies debido a que no fue posible obtener la información necesaria de todos los ejemplares. 
Por ejemplo, I. decemcornuta es una especie endémica de México, con muy pocos registros en el país. A pesar de no contar con datos de recolecta se incluyó en la estimación de la diversidad, ya que se sabe que el ejemplar consultado es del municipio de Coalcomán, pero no fue posible representarla en el mapa con la exactitud con la que se requería para el análisis.

Relación con factores ambientales. Los registros de la distribución altitudinal muestran que el intervalo en el que se desarrolla este género es muy amplio, ya que abarca desde el nivel del mar hasta $3000 \mathrm{~m}$. Sin embargo, las especies se presentan más frecuentemente entre 0 y $299 \mathrm{~m}$ en la zona sur del estado cercana a la costa y entre los 1800 y 2099 m (Fig. 4A), que corresponden principalmente a la parte norte. Las exploraciones realizadas para el presente estudio concuerdan con la poca riqueza y abundancia de especies entre ambos intervalos altitudinales (300-1 799 $\mathrm{m})$. Esta tendencia altitudinal es probable que se relacione con los 2 centros de diversidad encontrados, en el suroeste (0-299 m) y noreste (1 800-2 $099 \mathrm{~m})$. La elevada riqueza encontrada a bajas altitudes, difiere de lo registrado para Salvia, que presenta la mayor riqueza entre los 2001-2 500 m (Cornejo-Tenorio e Ibarra-Manríquez, 2011). Por otra parte, el haber encontrado mayor riqueza de especies en el bosque tropical caducifolio, coincide con lo encontrado en el componente arbóreo y pone de manifiesto la necesidad de continuar con los esfuerzos de conservación de este tipo de vegetación que representa la mayor cobertura en el estado (Cué Bär et al., 2006a, b; Velázquez et al., 2009). Además, se encontró un alto número de especies en el pastizal secundario, lo que concuerda con su caracterización de especies ruderales, arvenses e incluso indicadoras de disturbio (Carranza, 2004). Por último, se encontró una amplia distribución de las especies en 12 de los 14 tipos de suelo registrados para el estado. El mayor número de especies se encontró en el litosol, vertisol y luvisol que son los suelos que cubren la mayor superficie en el área de estudio (Durán y Sevilla, 2003), por lo que el tipo de suelo a la escala analizada no parece relacionarse de manera directa con la presencia de las especies de Ipomoea.

Finalmente, el hecho de que los registros de las 2 especies endémicas se encuentren en un área muy pequeña (Cuadro 1, Fig. 5) las hace particularmente vulnerables, y aumenta las probabilidades de su extinción si se continúa con la destrucción del hábitat y cambio del uso de suelo. Es urgente realizar esfuerzos de conservación en Michoacán, para lo cual debe considerarse la propuesta de Velázquez et al. (2009), quienes tomando en cuenta la opinión de diversos sectores y a partir de la riqueza y endemismos de las especies arbóreas, proponen sitios de conservación, uno de los cuales coincide con la distribución conocida de las 2 especies endémicas aquí registradas (Fig. 5). En el

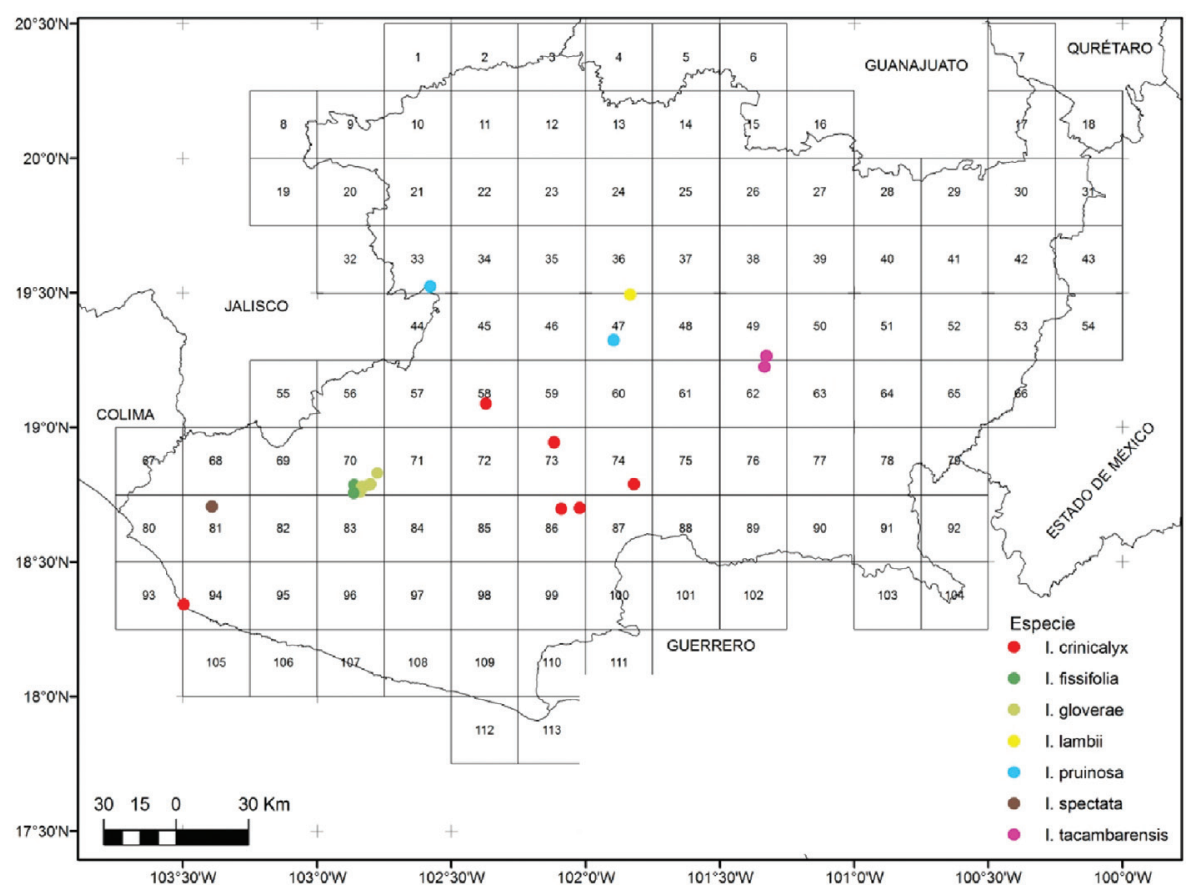

Figura 5. Mapa del estado de Michoacán con la retícula de $15^{\prime} \times 15^{\prime}$ y los registros de las 2 especies endémicas (I. fissifolia e $I$. gloverae), así como los registros de las especies de distribución restringida a Michoacán y otro estado de la República Mexicana. 
caso de I. fissifolia, no se sabe si en la actualidad existen poblaciones viables, debido a que el último registro es de hace 20 años. De acuerdo con los criterios de la NOM059-2001, I. gloverae puede considerarse en la categoría de especie amenazada.

Los resultados de este estudio confirman la relevancia florística de Michoacán y aportan información que deberá considerarse en las futuras propuestas para reservas de áreas naturales protegidas del estado.

\section{Agradecimientos}

A Ivonne Silva y Julio Valdez, por su valiosa ayuda en campo. Julio Valdez proporcionó la mayoría de las fotos de Ipomoea y Hernán Alvarado ayudó en la edición de las mismas. Al COECyT Michoacán, por la beca otorgada a J. Alcántar durante la realización de este estudio. D. Ávila y F. Rosas hicieron valiosos comentarios en versiones previas del trabajo. El Dr. A. McDonald y dos revisores anónimos aportaron valiosas sugerencias para mejorar el contenido del manuscrito. Por la misma razón se agradecen los comentarios del Dr. Guillermo Ibarra Manríquez en su calidad de editor asociado.

\section{Literatura citada}

Antaramián, H. E. 2005. Clima. In La biodiversidad en Michoacán: estudio de estado, L. E. Villaseñor (ed.). Comisión Nacional para el Conocimiento y Uso de la Biodiversidad/Secretaría de Urbanismo y Medio Ambiente/ Universidad Michoacana de San Nicolás de Hidalgo, Morelia, Michoacán. p. 25-28.

Austin, D. F. y R. A. Pedraza. 1983. Los géneros de Convolvulaceae en México. Boletín de la Sociedad Botánica de México 44:3-16.

Austin, D. F. y Z. Huáman. 1996. A synopsis of Ipomoea (Convolvulaceae) in the Americas. Taxon 45:3-38.

Carranza, E. 1998. Las especies del género Ipomoea L. (Convolvulaceae) en el estado de Guanajuato, México: taxonomía, distribución geográfica y ecológica, usos y conservación. Tesis, maestría Facultad de Biología, Universidad Michoacana de San Nicolás de Hidalgo, Morelia, Michoacán. 137 p.

Carranza, E. 2004. Análisis taxonómico y fitogeográfico del género Ipomoea L. (Convolvulaceae) en la flora del Bajío y de regiones adyacentes, México. Tesis, doctorado Facultad de Ciencias Naturales, Universidad Autónoma de Querétaro, Querétaro. 247 p.

Carranza, E. 2005a. Angiospermas. In La biodiversidad en Michoacán: Estudio de estado. L. E. Villaseñor (ed.). CONABIO/ Secretaría de Urbanismo y Medio Ambiente/ Universidad Michoacana de San Nicolás de Hidalgo.
Morelia, Michoacán. p. 73-75.

Carranza, E. 2005b. Vegetación. In La biodiversidad en Michoacán: estudio de estado. L. E. Villaseñor (ed.) CONABIO/ Secretaría de Urbanismo y Medio Ambiente/ Universidad Michoacana de San Nicolás de Hidalgo, Morelia, Michoacán. p. 38-46.

Carranza, E. 2008. Diversidad del género Ipomoea L. (Convolvulaceae) en el estado de Michoacán, México. In Flora del Bajío y regiones adyacentes, Fascículo complementario XXIII:5-123.

Cornejo-Tenorio, G. y G. Ibarra-Manríquez. 2011. Diversidad y distribución del género Salvia (Lamiaceae) en Michoacán, México. Revista Mexicana de Biodiversidad 82:1279-1296. Cué Bär, E., J. L. Villaseñor, L. Arredondo, G. Cornejo y G. Ibarra. 2006a. La flora arbórea de Michoacán, México. Boletín de la Sociedad Botánica de México 78:47-48.

Cué-Bär, E. M., J. L. Villaseñor, J. J. Morrone y G. IbarraManríquez. 2006b. Identifying priority areas for conservation in Mexican tropical deciduous forest based on tree species. Interciencia 31:712-719.

Cuevas-Arias, C. T, O. Vargas y A. Rodríguez. 2008. Solanaceae diversity in the state of Jalisco México. Revista Mexicana de Biodiversidad 79:67-79.

Durán, C. V. y P. F. Sevilla. 2003. Atlas geográfico del estado de Michoacán. Universidad Michoacana de San Nicolás de Hidalgo/ El Colegio de Michoacán/ Secretaría de Educación en el Estado de Michoacán/ EDDISA, Morelia, Michoacán.

Espinosa, D. y S. Ocegueda. 2008. El conocimiento biogeográfico de las especies y su regionalización natural. In Capital natural de México, I. Conocimiento actual de la biodiversidad. CONABIO, México, D. F. p. 33-65.

García, G., O. Rosales, M. de la Cerda y M. Siqueiros. 1999. Listado florístico del estado de Aguascalientes. Scientiae Naturae 1:1-25.

García-Mendoza, A. y J. Meave (eds.). 2011. Diversidad florística de Oaxaca: de musgos a angiospermas (colecciones y lista de especies). Instituto de Biología, Universidad Nacional Autónoma de México, México, D. F. 351 p.

Hijmans, J. R., S. E. Cameron, J. L. Parra, P. Jones y A. Jarvis. 2005. Very high resolution interpolated climate surfaces for global land areas. International Journal of Climatology 25:1965-1978.

INEGI (Instituto Nacional de Estadística, Geografía e Informática). 2005. Marco geoestadístico municipal. II. Conteo de población y vivienda (MGM-II Conteo 2005). ver 1.0. http://antares.inegi.gob.mx/meta/mgm/mgm2005_ espanol.html; ultima consulta: 10.XII.2011.

INEGI (Instituto Nacional de Estadística, Geografía e Informática). 2010. Marco geostadístico nacional. Datos vectoriales, ver. 5.0. http://www.inegi.org.mx/geo/ contenidos/geoestadistica/M_Geoestadistico.aspx; última 
consulta: 10.XII.2011.

Lindenmayer D. B., R. B Cunningham, H. A. Nix, M.T. Tanton y A. P. Smith. 1991. Predicting the abundance of hollowbearing trees in montane forest of southeastern Australia. Australian Journal of Ecology 16:91-98.

McDonald, A. 1991. Origin and diversity of Mexican Convolvulaceae. Anales del Instituto de Biología, Universidad Nacional Autónoma de México, Serie Botánica 62:65-82.

McDonald, A. 1994. Convolvulaceae II. Flora de Veracruz, fascículo 77. Instituto de Ecología/ University of California, Xalapa, Veracruz. p. 1-133.

McPherson, G. D. 1980. Eight new species of Ipomoea and Quamoclit from Mexico. Contributions from the University of Michigan Herbarium 14:85-97.

Medina, C., F. Guevara-Féfer, M. A. Martínez, P. Silva-Sáenz y M. A. Chávez-Carbajal. 2000. Estudio florístico en el área de la comunidad indígena de Nuevo San Juan Parangaricutiro, Michoacán, México. Acta Botanica Mexicana 52:5-41.

Murguía, G., J. Márquez, G. Laguna y M. Ponce. 1995. Estudio de frutos y semillas de Ipomoea teotitlanica McPherson (Convolvulaceae). Acta Botanica Mexicana 32:69-77.

Murguía, M. y F. Rojas. 2001. Biogeografía cuantitativa. In Introducción a la biogeografía en Latinoamérica: teorías, conceptos, métodos y aplicaciones, B. J. Llorente y J. J. Morrone (eds.). Las Prensas de Ciencias, Universidad Nacional Autónoma de México, México, D. F. p. 39-47.

Ramírez, D. R., O. P. Vargas, I. J. N. Arreola, M. M. Cedano, R. T. González, M. L. V. González, J. A. R. Pérez, A. C. Rodríguez, J. J. D. Reynoso, L. M. P. Villarreal y J. L. Villaseñor. 2010. Catálogo de plantas vasculares de Jalisco. Universidad de Guadalajara/ Sociedad Botánica de México/ Universidad Autónoma Metropolitana, México, D. F. 143 p.
Ramos-Vizcaíno, I., S. Guerrero-Vázquez y F. M. HuertaMartínez. 2007. Patrones de distribución geográfica de los mamíferos de Jalisco, México. Revista Mexicana de Biodiversidad 78:175-189.

Rzedowski, J. 1978. Vegetación de México. Limusa, México, D. F. 432 p.

Rzedowski, J. 1991. Diversidad y orígenes de la flora fanerogámica de México. Acta Botanica Mexicana 14:3-21.

Rzedowski, J., L. R. Medina y G. Calderon. 2005. Inventario del conocimiento taxonómico, así como de la diversidad y del endemismo regionales de las especies mexicanas de Bursera (Burseraceae). Acta Botanica Mexicana 70:85-111.

Trejo, I. 2005. Análisis de la diversidad de la selva baja caducifolia en México. In Sobre diversidad biológica: el significado de las diversidades alfa, beta y gamma. Monografías Tercer Milenio, G. Halffter, J. Soberón, P. Koleff y A. Melic (eds.). Sociedad Entomológica Aragonesa, Zaragoza. p. 111-122.

Valencia, A. S. 2004. Diversidad del género Quercus (Fagaceae) en México. Boletín de la Sociedad Botánica de México 75:33-53.

Velázquez, A., E. M. Cué-Bär, A. Larrazabal, N. Sosa, J. L. Villaseñor y G. Ibarra-Manríquez. 2009. Building participatory landscape-based conservation alternatives: a case of study of Michoacán, Mexico. Applied Geography 29:513-526.

Villaseñor, J. L. 2003. Diversidad y distribución de las Magnoliophyta de México. Interciencia 28:160-167.

Villaseñor, J. L. 2004. Los géneros de plantas vasculares de la flora de México. Boletín de la Sociedad Botánica de México 75:105-135.

Villaseñor, J. L., G. Ibarra-Manríquez, J. A. Meave y E. Ortiz. 2005. Higher taxa as surrogates of plant biodiversity in a megadiverse country. Conservation Biology 19:232-238.

Apéndice. Especies de Ipomoea presentes en Michoacán y condiciones en las que se localizan. Fila de títulos. $R F$, región fisiográfica; $A L T$, altitud; $P P$, precipitación; TEM, temperatura. Columnas RF y TV. BE, bosque de encino; BM, bosque mesófilo de montaña; $\mathrm{BP}$, bosque de pino; BPE, bosque de pino-encino; BTC, bosque tropical caducifolio; BTS, bosque tropical subcaducifolio; EN, Eje Neovolcánico; MS, matorral subtropical; PS, pastizal secundario; SMS, Sierra Madre del Sur; VDC, vegetación de dunas costeras; ZL, zona limítrofe. Superíndices. A, arbórea; Ar, arbustiva; HE, herbácea erecta; RH, rastrera herbácea; TH, trepadora herbácea; TL, trepadora leñosa. En negritas: número de registros.

\begin{tabular}{|c|c|c|c|c|c|}
\hline Especie & $R F$ & $T V$ & $A L T(m)$ & $P P(m m)$ & $\operatorname{TEM}\left({ }^{\circ} \mathrm{C}\right)$ \\
\hline Ipomoea alba $\mathrm{L} .{ }^{(\mathrm{RH}, \mathrm{TH})} 4$ & SMS, EN & PS & $0-2000$ & $676-935$ & $17-28$ \\
\hline I. ampullacea Fernald ${ }^{(\mathrm{TH})} 3$ & SMS & BTC, BTS, BE & $100-1300$ & $1085-1320$ & $22-26$ \\
\hline I. arborescens (Humb. et Bompl. ex Willd.) G. Don ${ }^{(\mathrm{A}, \mathrm{Ar}) 6}$ & EN, SMS & BTC & $100-700$ & $980-1088$ & $22-25$ \\
\hline I. barbatisepala A. Gray ${ }^{(\mathrm{TH})} \mathbf{1}$ & ZL & BTC & 300 & 692 & 28 \\
\hline I. batatas (L.) Lam. ${ }^{(\mathrm{TH})} 3$ & SMS, ZL & PS & $100-1600$ & 926 & 26 \\
\hline I. batatoides Choisy ${ }^{(\mathrm{TL}) 2}$ & SMS, ZL & BTS & $100-150$ & $946-1115$ & $25-26$ \\
\hline I. bracteata $\mathrm{Cav}{ }^{(\mathrm{TL})} 16$ & EN, SMS & BTC & $0-100$ & $616-1079$ & $17-29$ \\
\hline I. capillacea (Kunth) G. Don ${ }^{(\mathrm{RH})} 20$ & EN & BE, BPE, PS, MS & $1900-2400$ & $821-1413$ & $15-19$ \\
\hline
\end{tabular}


Apéndice. Continúa.

\begin{tabular}{|c|c|c|c|c|c|}
\hline Especie & $R F$ & $T V$ & $A L T(m)$ & $P P(m m)$ & $\operatorname{TEM}\left({ }^{\circ} \mathrm{C}\right)$ \\
\hline I. cardiophylla A. Gray ${ }^{(\mathrm{TH})} 17$ & EN & BPE, BE, PS & $1600-1850$ & $684-778$ & $18-19$ \\
\hline I. clavata (G. Don) Ooststr. ex J.F. Macbr. ${ }^{(\mathrm{TL})} 1$ & SMS & BTS & $100-150$ & 948 & 26 \\
\hline I. costellata Torr. ${ }^{(\mathrm{RH})} 9$ & EN, ZL & BTC, MS & $300-2100$ & $691-1045$ & $17-29$ \\
\hline I. crinicalyx $\mathrm{S}$. Moore ${ }^{(\mathrm{RH})} 6$ & SMS, ZL & BTC, PS & $50-1000$ & $602-889$ & $25-28$ \\
\hline I. cristulata Hallier $\mathrm{f}{ }^{(\mathrm{TH})} 15$ & EN & BPE, BE, MS & $1700-2100$ & $686-1024$ & $15-20$ \\
\hline I. cuprinacoma E. Carranza et J. A. McDonald ${ }^{(\mathrm{TL}) 5}$ & EN, SMS & BTC, BTS & $700-2000$ & $843-992$ & $19-23$ \\
\hline I. decasperma Hallier $\mathrm{f}$. ${ }^{(\mathrm{RH}, \mathrm{TH})} \mathbf{2 0}$ & EN, SMS & BTC, BPE, BE & $1300-2700$ & $750-1349$ & $13-20$ \\
\hline I decemcornuta O'Donell ${ }^{(\mathrm{RH})} 1$ & SMS & & $1000-1200$ & & \\
\hline I. dimorphophylla Greenm. ${ }^{(\mathrm{RH})} 2$ & SMS, ZL & BTC & $400-900$ & $1004-1087$ & $24-27$ \\
\hline I. dumetorum Willd. ex Roem. et Schult. ${ }^{(\mathrm{RH}, \mathrm{TH}) 9}$ & EN & BP, BE, BPE & $2000-2500$ & $825-1144$ & $13-17$ \\
\hline I. dumosa (Benth.) L.O. Williams ${ }^{(\mathrm{TH}, \mathrm{TL}) 5}$ & EN, ZL & BPE, BE & $1600-2300$ & $883-1208$ & $14-20$ \\
\hline I. elongata Choisy ${ }^{(\mathrm{RH}, \mathrm{TH}) 3}$ & EN & $\mathrm{BPE}, \mathrm{BE}$ & $1600-2000$ & $1064-1413$ & $18-20$ \\
\hline I. fissifolia (McPherson) J.E. Ecknwalder ${ }^{(\mathrm{TL})} 2$ & SMS & BTC & 1450 & 1323 & 20 \\
\hline I. funis Schltdl. et Cham. var. langlassei (House) O’Donell ${ }^{(\mathrm{TH}, \mathrm{TL})} 9$ & EN, SMS & BP, BPE, BM & $1800-2450$ & $1015-1348$ & $14-17$ \\
\hline I. gloverae J.A. McDonald ${ }^{(\mathrm{RH}) 6}$ & SMS & BTC & $1000-1450$ & $919-1323$ & $20-25$ \\
\hline I. hartwegii Benth ${ }^{(\mathrm{RH})} 20$ & EN & MS, PS & $1700-2450$ & 714-1015 & $16-19$ \\
\hline I. hastigera Kunth ${ }^{(\mathrm{TL}) 4}$ & SMS & $\mathrm{BPE}$ & $1750-2100$ & $1230-1359$ & $17-22$ \\
\hline I. hederacea (L.) Jacq. ${ }^{(\mathrm{TH}) 3}$ & SMS & BTC & $20-300$ & $884-1159$ & $25-27$ \\
\hline I. hederifolia $\mathrm{L} .^{(\mathrm{TH})} 12$ & EN, SMS & BTC, PS & $50-1850$ & $646-1352$ & $17-29$ \\
\hline I. indica (Burm. f.) Merr. ${ }^{(\mathrm{TH}) 6}$ & SMS & BTC, BPE & $50-2150$ & $839-1294$ & $17-27$ \\
\hline I. lambii Fernald ${ }^{(\mathrm{TH}) 1}$ & EN & $\mathrm{BPE}$ & 2200 & 1106 & 16 \\
\hline I. leucantha Jacq. ${ }^{(\mathrm{TH})} 1$ & EN & PS & 1900 & 793 & 18 \\
\hline I. lindenii M. Martens et Galeotti ${ }^{(\mathrm{TL}) 1}$ & SMS & STS & $350-600$ & 883 & 25 \\
\hline I. lobata (Cerv.) Thell. ${ }^{(\mathrm{TH})} 6$ & EN, ZL & BTC & $900-1700$ & 712 & $18-27$ \\
\hline I. lottie J.A. McDonald ${ }^{(\mathrm{TH}, \mathrm{TL}) 2}$ & SMS & BTC & $50-300$ & $1070-1159$ & $25-26$ \\
\hline I. madrensis $\mathrm{S}$. Watson ${ }^{(\mathrm{HE}) 5}$ & EN & $\mathrm{BPE}, \mathrm{BE}$ & $2100-2650$ & $825-1241$ & $13-17$ \\
\hline I. mairetii Choisy ${ }^{(\mathrm{TL}) 3}$ & SMS & BTC & $500-1400$ & $883-1337$ & $20-25$ \\
\hline I. meyeri (Spreng.) G. Don ${ }^{(\mathrm{TH}) 3}$ & SMS & BTS & $50-700$ & $952-1083$ & 26 \\
\hline I. microsepala Benth. ${ }^{(\mathrm{TL}) 2}$ & SMS & BTC, PS & $50-1200$ & $717-1008$ & $26-28$ \\
\hline I. minutiflora (M. Martens etGaleotti) House ${ }^{(\mathrm{RH}) 4}$ & SMS, ZL & BTC & $50-900$ & $868-1060$ & $26-27$ \\
\hline I. muricata (L.) Jacq. ${ }^{(\mathrm{TH})} 3$ & SMS, ZL & BTC, BE & $100-1400$ & $787-1320$ & $21-28$ \\
\hline I. murucoides Roem. et Schult. ${ }^{(\mathrm{A}) 44}$ & $\mathrm{EN}$ & BTC, BE, MS, PS & $1550-2400$ & $704-1080$ & $15-20$ \\
\hline I. neei (Spreng.) O’Donell( ${ }^{\mathrm{TL})} 9$ & EN, SMS & BTC, BM, PS & $800-1600$ & 803-1376 & $19-23$ \\
\hline I. neurocephala Hallier f. ${ }^{(\mathrm{TL})} 7$ & SMS, ZL & BTC & $650-1600$ & $919-1352$ & $20-26$ \\
\hline I. nil (L.) Roth ${ }^{(\mathrm{RH}, \mathrm{TH}) 7}$ & SMS, ZL & BTC & $50-1000$ & $707-896$ & $23-29$ \\
\hline I. oocarpa Benth. $^{(\mathrm{TH})} 3$ & SMS, ZL & BTC & $200-1000$ & $842-1291$ & $22-25$ \\
\hline I. orizabensis (G. Pelletan) Ledeb. ex Steud. var. orizabensis ${ }^{(\mathrm{TH})} 60$ & EN, SMS & BP, BPE, BE, PS & $1000-3000$ & $718-1271$ & $13-20$ \\
\hline I. orizabensis var. novogaliciana J.A. McDonald ${ }^{(\mathrm{TH}) 4}$ & EN & $\mathrm{BPE}$ & $1700-2100$ & $704-1080$ & $14-20$ \\
\hline I. parasitica (Kunth) G. Don ${ }^{(\mathrm{TH}) 10}$ & SMS, ZL & BTC, BE & $500-1400$ & $801-1306$ & $23-28$ \\
\hline I. pauciflora M. Martens et Galeotti subsp. pauciflora ${ }^{(\mathrm{A}, \mathrm{Ar})} 3$ & SMS, ZL & BTC & $300-1200$ & $720-974$ & $24-27$ \\
\hline I. pedicellaris Benth. ${ }^{(\mathrm{RH}, \mathrm{TH})} 2$ & SMS, ZL & BTC & $100-1300$ & $960-980$ & $26-28$ \\
\hline
\end{tabular}


Apéndice. Continúa.

\begin{tabular}{|c|c|c|c|c|c|}
\hline Especie & $R F$ & $T V$ & $A L T(m)$ & $P P(\mathrm{~mm})$ & $\operatorname{TEM}\left({ }^{\circ} \mathrm{C}\right)$ \\
\hline I. pes-caprae (L.) R. Br. ${ }^{(\mathrm{RH})} 4$ & SMS & VDC & $0-50$ & $894-1070$ & $26-27$ \\
\hline I. plummerae A. Gray ${ }^{(\mathrm{RH})} 16$ & EN, SMS & BP, BPE, BE, PS & $2000-2400$ & $890-1326$ & $15-17$ \\
\hline I. praecana House $\mathrm{e}^{(\mathrm{TL}) 5}$ & SMS & BTC & $50-900$ & $686-1159$ & $25-28$ \\
\hline I. aff. proxima (M. Martens et Galeotti) Hemsl. ${ }^{(\mathrm{TH})} 1$ & ZL & BTC & 700 & 1180 & 26 \\
\hline I. pruinosa $\mathrm{McPherson}{ }^{(\mathrm{TH}, \mathrm{TL}) 3}$ & ZL & BTC, BE & $400-1200$ & $1055-1086$ & $22-23$ \\
\hline I. $p_{\text {seudoracemosa }}$ McPherson ${ }^{(\mathrm{TH}, \mathrm{TL}) 6}$ & ZL & BTC & $300-700$ & $651-936$ & $25-29$ \\
\hline I. pubescens Lam. ${ }^{(\mathrm{RH}, \mathrm{TH})} 3$ & $\mathrm{EN}$ & PS & $2000-2350$ & 808-990 & $15-18$ \\
\hline I. puncticulata Benth..$^{(\mathrm{RH}, \mathrm{TH})} 5$ & SMS & BTC, BTS & $150-1400$ & $717-1320$ & $21-29$ \\
\hline I. purpurea (L.) Roth ${ }^{(\mathrm{RH}, \mathrm{TH})} 46$ & EN, SMS & BTC, BE, BPE, MS, PS & $850-2700$ & $685-1593$ & $13-20$ \\
\hline I. quamoclit $\mathrm{L}$. $^{(\mathrm{H})} 3$ & SMS, ZL & BTC & $0-300$ & $1045-1083$ & $26-27$ \\
\hline I. robinsonii House $\mathrm{e}^{(\mathrm{TH}) 5}$ & SMS, ZL & BTC & $200-1000$ & $829-1354$ & $15-27$ \\
\hline I. santillanii $\mathrm{O}^{\prime}$ Donell ${ }^{(\mathrm{TH})} 7$ & EN, SMS & BTC & $1450-2400$ & $850-1370$ & $16-20$ \\
\hline I. seducta House $\mathrm{e}^{(\mathrm{TH}) 3}$ & SMS & $\mathrm{BTC}$ & $600-1800$ & $958-1336$ & $22-24$ \\
\hline I. setosa $\mathrm{Ker} G a w l^{(\mathrm{TH}, \mathrm{TL})} 4$ & SMS & BTS & $50-600$ & $717-1109$ & $26-29$ \\
\hline I. simulans D. Hanb. ${ }^{(\mathrm{TH}) 2}$ & EN & PS & $2300-2950$ & $921-922$ & 15 \\
\hline I. spectata J.A. McDonald ${ }^{(\mathrm{TH})} \mathbf{1}$ & SMS & BPE & $1150-1500$ & 1358 & 20 \\
\hline I. stans Cav. ${ }^{(\mathrm{RH}, \mathrm{TH})} 11$ & $\mathrm{EN}$ & BE, MS, PS & $1450-2750$ & $715-979$ & $16-18$ \\
\hline I. suaveolens (M. Martens et Galeotti) Hemsl. ${ }^{(\mathrm{TH}, \mathrm{TL}) 4}$ & SMS, ZL & BTC & $50-1250$ & $1038-1272$ & $23-26$ \\
\hline I. suffulta (Kunth) G. Don ${ }^{(\mathrm{RH}, \mathrm{TH})} 5$ & EN, SMS & BTC, BE, BPE & $850-1800$ & $842-1214$ & $18-26$ \\
\hline I. tacambarensis E. Carranza ${ }^{(\mathrm{HE}) 3}$ & $\mathrm{ZL}$ & $\mathrm{BPE}$ & $1200-1850$ & $1135-1166$ & $18-22$ \\
\hline I. ternifolia Cav. var. ternifolia ${ }^{(\mathrm{HE}) 7}$ & EN, SMS & BTC, PS & $50-1850$ & $694-947$ & $18-28$ \\
\hline I. tiliacea (Willd.) Choisy ${ }^{(\mathrm{RH}, \mathrm{TH})} \mathbf{5}$ & SMS & BTC, BTS & $50-550$ & $717-1341$ & $19-29$ \\
\hline I. tricolor $\mathrm{Cav}^{(\mathrm{TH}) 5}$ & EN & PS & $300-2300$ & $682--800$ & $16-18$ \\
\hline I. trifida (Kunth) G. Don ${ }^{(\mathrm{TH})} 7$ & SMS, ZL & BTC, PS & $0-1500$ & $805-1202$ & $22-27$ \\
\hline I. triloba $\mathrm{L}^{(\mathrm{TH})} 9$ & EN, SMS & BTC, PS & $50-1800$ & 603-1079 & $19-29$ \\
\hline I. urbinei House $\mathrm{T}^{(\mathrm{TL}) 7}$ & SMS, ZL & $\mathrm{BP}, \mathrm{BPE}, \mathrm{BE}$ & $1200-1700$ & $1227-1488$ & $17-23$ \\
\hline I. wolcottiana Rose var. wolcottiana ${ }^{(\mathrm{A}) 3}$ & SMS & BTC & $50-700$ & $1074-1159$ & $25-26$ \\
\hline
\end{tabular}

